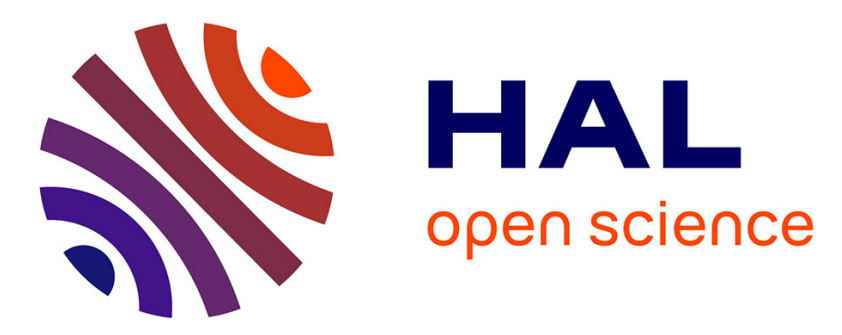

\title{
State and input observability for structured linear systems: A graph-theoretic approach
}

\author{
Taha Boukhobza, Frédéric Hamelin, Sinuhé Martinez-Martinez
}

\section{To cite this version:}

Taha Boukhobza, Frédéric Hamelin, Sinuhé Martinez-Martinez. State and input observability for structured linear systems: A graph-theoretic approach. Automatica, 2007, 43 (7), pp.1204-1210. 10.1016/j.automatica.2006.12.004 . hal-00152192

\section{HAL Id: hal-00152192 \\ https://hal.science/hal-00152192}

Submitted on 6 Jun 2007

HAL is a multi-disciplinary open access archive for the deposit and dissemination of scientific research documents, whether they are published or not. The documents may come from teaching and research institutions in France or abroad, or from public or private research centers.
L'archive ouverte pluridisciplinaire HAL, est destinée au dépôt et à la diffusion de documents scientifiques de niveau recherche, publiés ou non, émanant des établissements d'enseignement et de recherche français ou étrangers, des laboratoires publics ou privés. 


\title{
State and input observability for structured linear systems: A graph-theoretic approach
}

\author{
T. Boukhobza, F. Hamelin and S. Martinez-Martinez \\ Centre de Recherche en Automatique de Nancy, Nancy-University, CNRS UMR 7039, BP 239, 54506 Vandouvre, \\ France.Phone: 33383684 464, Fax: 33383684 462, email: taha.boukhobza@cran.uhp-nancy.fr
}

\begin{abstract}
This paper deals with the state and input observability analysis for structured linear systems with unknown inputs. The proposed method is based on a graph-theoretic approach and assumes only the knowledge of the system's structure. Using a particular decomposition of the systems into two subsystems, we express, in simple graphic terms, necessary and sufficient conditions for the generic state and input observability. These conditions are easy to check because they are based on comparison of integers and on finding particular subgraphs in a digraph. Therefore, our approach is suited to study large scale systems.
\end{abstract}

Key words: Graph theory, structured systems, state and input generic observability.

\section{Introduction}

The problem of estimating the state and the unknown input is of great interest mainly in control law synthesis, fault detection and isolation, fault tolerant control, supervision and so on. In this respect, many works (Chu, 2000; Chu and Mehrmann, 1999; Hou et al., 1999; Trin and Ha, 2000; Tsui, 1996) are focused on the design of state observers for linear systems subject to unknown inputs. Otherwise, the issue of simultaneously observing the whole state and the unknown input has been investigated in (Hou and Müller, 1992; Koenig, 2005).

In most cases, the studies on the state and input observability deal with algebraic and geometric tools (Basile and Marro, 1973; Hou and Müller, 1999; Hou and Patton, 1998; Trentelman et al., 2001). The use of such tools requires the exact knowledge of the state space matrices characterizing the system's model. However, in many modeling problems, these matrices have a number of fixed zero entries determined by the physical laws while the remaining entries are not precisely known. To study the properties of these systems in spite of poor knowledge we have on them, the idea is that we only keep the zero/non-zero entries in the state space matrices. Thus, we consider models where the fixed zeros are conserved while the non-zero entries are replaced by free parameters. There is a huge amount of interesting works in the literature using this kind of models called structured models. The study of such systems requires a low computational burden which allows one to deal with large scale systems. Many studies on structured systems are related to the graph-theoretic approach to analyse some system properties such as controllability, observability or the solvability of several classical control problems including disturbance rejection, input-output decoupling, ... (Dion et al., 2003). It results from these works that the graph-theoretic approach provides simple and elegant solutions.
However, the well-known graphic observability conditions for linear structured systems recalled in (Dion et al., 2003) cannot be applied to systems with unknown inputs. More recently, in (Boukhobza et al., 2006) authors express in graphic terms necessary and sufficient conditions for the observability of general descriptor systems. Nevertheless, these conditions are quite complicated.

In this context, the purpose of this paper is to use a graphtheoretic approach for providing necessary and sufficient conditions for the state and input observability for structured linear systems.

The paper is organised as follows: after Section 2, which is devoted to the problem formulation, a digraph representation of structured systems is given in Section 3. The main result is enounced in Section 4. Finally, some concluding remarks are made.

\section{Problem formulation}

In this paper, we treat numerically non-specified systems on the form:

$$
\Sigma:\left\{\begin{array}{l}
\dot{x}(t)=A x(t)+B u(t) \\
y(t)=C x(t)+D u(t)
\end{array}\right.
$$

where $x \in \mathbb{R}^{n}, u \in \mathbb{R}^{q}$ and $y \in \mathbb{R}^{p}$ are respectively the state vector, the unknown input vector and the output vector. We assume that only the zero/nonzero structure of $A, B, C$ and $D$ is known. This means that, to each entry in these matrices, we only know whether its value is fixed to zero, in which case we call it a fixed zero, or that it has an unknown real value, in which case we call the entry a free parameter. In a structured system with $h$ nonzero entries in $A, B, C$ and $D$, we can parameterize these nonzero entries by scalar real (nonzero) parameters $\lambda_{i}, i=1, \ldots, h$ forming a parameter 
vector $\Lambda=\left(\lambda_{1}, \ldots, \lambda_{h}\right)^{T} \in \mathbb{R}^{h}$. We denote by $A^{\lambda}, B^{\lambda}, C^{\lambda}$ and $D^{\lambda}$ respectively the matrices obtained by replacing the nonzeros in $A, B, C$ and $D$ by the corresponding parameters $\lambda_{i}, i=1, \ldots, h$, and we denote

$$
\Sigma_{\Lambda}:\left\{\begin{array}{l}
\dot{x}(t)=A^{\lambda} x(t)+B^{\lambda} u(t) \\
y(t)=C^{\lambda} x(t)+D^{\lambda} u(t)
\end{array}\right.
$$

If all parameters $\lambda_{i}$ are numerically fixed, we obtain a socalled admissible realization of structured system $\Sigma_{\Lambda}$. More precisely, a realization of $\Sigma_{\Lambda}$ is a linear system $\Sigma$ which has no indeterminate parameters and has the same structure than $\Sigma_{\Lambda}$ i.e. $A(i, j)=0 \Leftrightarrow A^{\lambda}(i, j)=0, B(i, j)=$ $0 \Leftrightarrow B^{\lambda}(i, j)=0, C(i, j)=0 \Leftrightarrow C^{\lambda}(i, j)=0$ and $D(i, j)=0 \Leftrightarrow D^{\lambda}(i, j)=0$.

We say that a property is true generically (van der Woude, 2000) if it is true for almost all the realizations of structured system $\Sigma_{\Lambda}$. Here, "for almost all the realizations" is to be understood (Dion et al., 2003; van der Woude, 2000) as "for all parameter values $\left(\Lambda \in \mathbb{R}^{h}\right)$ except for those in some proper algebraic variety in the parameter space". The proper algebraic variety for which the property is not true is the zero set of some nontrivial polynomial with real coefficients in the $h$ system parameters $\lambda_{1}, \lambda_{2}, \ldots, \lambda_{h}$ or equivalently it is an algebraic variety which has Lebesgue measure zero (Reinschke, 1988).

In this paper, we study the generic state and input observability for structured system $\Sigma_{\Lambda}$. This notion is related to the strong observability and the left invertibility (Trentelman et al., 2001). Let us recall the definition of the generic state and input observability:

Definition 1 Consider structured system $\Sigma_{\Lambda}$, we say that $\Sigma_{\Lambda}$ is generically state and input observable if and only if it is generically strongly observable and left invertible.

In other words, system $\Sigma_{\Lambda}$ is generically state and input observable iff, for almost all realizations of $\Sigma_{\Lambda}$, for all initial state $x_{0}$ and for every input function $u(t), y(t)=0$ for $t \geq 0$ implies $x(t)=0$ for $t \geq 0$ and $u(t)=0$ for $t>0$. Roughly speaking, generic state and input observability means that a change in input or initial state can be reflected in a change of measurements.

Necessary and sufficient conditions for the state and input observability of structured system $\Sigma_{\Lambda}$ can be deduced from the ones provided in (Trentelman et al., 2001) or from the conditions of the right-hand side observability of a descriptor system (Hou and Müller, 1999):

Theorem 2 Consider structured system $\Sigma_{\Lambda}$ and let us denote $P(s)=\left(\begin{array}{cc}A^{\lambda}-s I_{n} & B^{\lambda} \\ C^{\lambda} & D^{\lambda}\end{array}\right)$. Structured system $\Sigma_{\Lambda}$ is generically state and input observable iff $\forall s \in \mathbb{C}$ $g \_r a n k(P(s))=n+g \_r a n k\left(\begin{array}{c}B^{\lambda} \\ D^{\lambda}\end{array}\right)=n+q$.

Assume that system $\Sigma_{\Lambda}$ is numerically specified. Regarding $P(s)$ as a rational matrix, we call its rank the normal-rank (van der Woude, 2000) and we denote this normal rank by $n$-rank $(P(s))$. Thus, for each realization of system $\Sigma_{\Lambda}$, we can compute the $n$-rank of $P(s)$. This rank will have the same value for almost all parameter values $\lambda \in \mathbb{R}^{h}$ (Reinschke, 1988; van der Woude, 2000). This so-called generic $n$-rank of $P(s)$ will be denoted by $g \_n$-rank $(P(s))$. Generic rank of matrix $P(s)$, denoted $g \_\operatorname{rank}(P(s))$, is quite different as it depends on $s$. Hence, $g \_\operatorname{rank}(P(s))=r, \forall s \in \mathbb{C}$ means that for almost all parameter values $\lambda \in \mathbb{R}^{h}, \operatorname{rank}(P(s))=r, \forall s \in \mathbb{C}$.

The aim of the paper is to give graphical conditions to analyse the question whether or not structured system $\Sigma_{\Lambda}$ is generically state and input observable. These conditions are equivalent to the ones of Theorem 2 but are quite easy to check since they are based on finding paths in a digraph.

\section{Graph representation of structured linear systems}

This section is devoted in a first stage to the definition of a digraph representing $\Sigma_{\Lambda}$. Next, we give some useful notations and definitions.

Digraph $\mathcal{G}\left(\Sigma_{\Lambda}\right)$ associated to $\Sigma_{\Lambda}$ is constituted by a vertex set $\mathcal{V}$ and an edge set $\mathcal{E}$ i.e. $\mathcal{G}\left(\Sigma_{\Lambda}\right)=(\mathcal{V}, \mathcal{E})$. More precisely, $\mathcal{V}=\mathbf{X} \cup \mathbf{Y} \cup \mathbf{U}$, where $\mathbf{X}=\left\{\mathbf{x}_{1}, \ldots, \mathbf{x}_{\mathbf{n}}\right\}$ is the set of state vertices, $\mathbf{Y}=\left\{\mathbf{y}_{\mathbf{1}}, \ldots, \mathbf{y}_{\mathbf{p}}\right\}$ is the set of output vertices and $\mathbf{U}=\left\{\mathbf{u}_{\mathbf{1}}, \ldots, \mathbf{u}_{\mathbf{q}}\right\}$ is the set of unknown input vertices. The edge set is $\mathcal{E}=A$-edges $\cup B$-edges $\cup C$-edges $\cup D$-edges, with $A$-edges $=\left\{\left(\mathbf{x}_{\mathbf{j}}, \mathbf{x}_{\mathbf{i}}\right) \mid A^{\lambda}(i, j) \neq 0\right\}$,

$B$-edges $=\left\{\left(\mathbf{u}_{\mathbf{j}}, \mathbf{x}_{\mathbf{i}}\right) \mid B^{\lambda}(i, j) \neq 0\right\}$,

$C$-edges $=\left\{\left(\mathbf{x}_{\mathbf{j}}, \mathbf{y}_{\mathbf{i}}\right) \mid C^{\lambda}(i, j) \neq 0\right\}$,

$D$-edges $=\left\{\left(\mathbf{u}_{\mathbf{j}}, \mathbf{y}_{\mathbf{i}}\right) \mid D^{\lambda}(i, j) \neq 0\right\}$.

Here, $M^{\lambda}(i, j)$ is the $(i, j)$ th element of matrix $M^{\lambda}$ and $\left(\mathbf{v}_{\mathbf{1}}, \mathbf{v}_{\mathbf{2}}\right)$ denotes a directed edge from vertex $\mathbf{v}_{\mathbf{1}} \in \mathcal{V}$ to vertex $\mathbf{v}_{\mathbf{2}} \in \mathcal{V}$.

Hereafter, we illustrate the proposed digraph representation with an example.

Example 3 To the system defined by the following matrices, we associate the digraph in Figure 1.

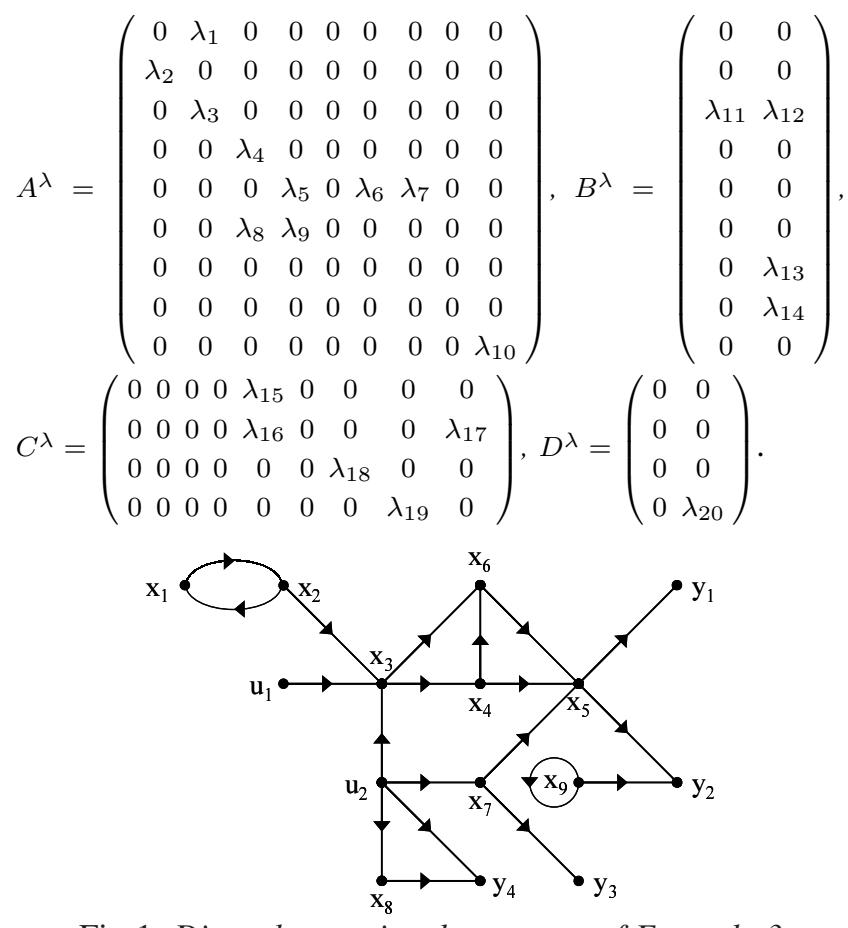

Fig 1: Digraph associated to system of Example 3 
Let us now give some useful definitions and notations.

- Two edges $e_{1}=\left(\mathbf{v}_{\mathbf{1}}, \mathbf{v}_{\mathbf{1}}^{\prime}\right)$ and $e_{2}=\left(\mathbf{v}_{\mathbf{2}}, \mathbf{v}_{\mathbf{2}}^{\prime}\right)$ are $v$ disjoint if $\mathbf{v}_{\mathbf{1}} \neq \mathbf{v}_{\mathbf{2}}$ and $\mathbf{v}_{\mathbf{1}}^{\prime} \neq \mathbf{v}_{\mathbf{2}}^{\prime}$. Note that $e_{1}$ and $e_{2}$ can be $v$-disjoint even if $\mathbf{v}_{\mathbf{1}}^{\prime}=\mathbf{v}_{\mathbf{2}}$ or $\mathbf{v}_{\mathbf{1}}=\mathbf{v}_{\mathbf{2}}^{\prime}$.

To illustrate the latter definition, note that in example 3 , $\left(\mathbf{x}_{5}, \mathbf{y}_{2}\right)$ and $\left(\mathbf{x}_{4}, \mathbf{x}_{5}\right)$ as well as $\left(\mathbf{x}_{2}, \mathbf{x}_{1}\right)$ and $\left(\mathbf{x}_{1}, \mathbf{x}_{2}\right)$ are $v$-disjoint. However, neither $\left(\mathbf{x}_{3}, \mathbf{x}_{6}\right)$ and $\left(\mathbf{x}_{4}, \mathbf{x}_{6}\right)$ nor $\left(\mathbf{x}_{\mathbf{7}}, \mathbf{y}_{\mathbf{3}}\right)$ and $\left(\mathbf{x}_{\mathbf{7}}, \mathbf{x}_{\mathbf{5}}\right)$ are $v$-disjoint. Some edges are $v$ disjoint if they are mutually $v$-disjoint.

- A subgraph $\mathcal{S}_{\mathcal{G}}$ of $\mathcal{G}\left(\Sigma_{\Lambda}\right)$ is defined by an edge subset $\mathcal{S}_{\mathcal{E}} \subseteq \mathcal{E}$ and a vertex subset $\mathcal{S}_{\mathcal{V}} \subseteq \mathcal{V}$ such that $\mathcal{S}_{\mathcal{V}}$ is constituted by the begin and the end vertices of all elements of $\mathcal{S}_{\mathcal{E}}$. We note $\mathcal{S}_{\mathcal{G}}=\left(\mathcal{S}_{\mathcal{V}}, \mathcal{S}_{\mathcal{E}}\right)$. A subgraph $\mathcal{S}_{\mathcal{G}}$ of $\mathcal{G}\left(\Sigma_{\Lambda}\right)$ is a $v$-disjoint subgraph if all its edges are $v$-disjoint. A subgraph $\mathcal{S}_{\mathcal{G}}=\left(\mathcal{S}_{\mathcal{V}}, \mathcal{S}_{\mathcal{E}}\right)$ of $\mathcal{G}\left(\Sigma_{\Lambda}\right)$ covers vertex $\mathbf{v}$ if $\exists e \in \mathcal{S}_{\mathcal{E}}$ such that $\mathbf{v}$ is the begin vertex of $e$.

- We denote path $P$ containing vertices $\mathbf{v}_{\mathbf{r}_{\mathbf{0}}}, \ldots, \mathbf{v}_{\mathbf{r}_{\mathbf{i}}}$ by $P=\mathbf{v}_{\mathbf{r}_{\mathbf{0}}} \rightarrow \mathbf{v}_{\mathbf{r}_{1}} \rightarrow \ldots \rightarrow \mathbf{v}_{\mathbf{r}_{\mathbf{i}}}$, where $\left(\mathbf{v}_{\mathbf{r}_{\mathbf{j}}}, \mathbf{v}_{\mathbf{r}_{\mathbf{j}+1}}\right) \in \mathcal{E}$ for $j=0,1 \ldots, i-1$. A cycle is a path of the form $\mathbf{v}_{\mathbf{r}_{0}} \rightarrow \mathbf{v}_{\mathbf{r}_{1}} \rightarrow \ldots \rightarrow \mathbf{v}_{\mathbf{r}_{\mathbf{i}}} \rightarrow \mathbf{v}_{\mathbf{r}_{0}}$, where all vertices $\mathbf{v}_{\mathbf{r}_{0}}$, $\mathbf{v}_{\mathbf{r}_{1}}, \ldots, \mathbf{v}_{\mathbf{r}_{i}}$ are distinct. Some paths are disjoint if they have no common vertex. Path $P$ is an $\mathbf{Y}$-topped path if its end vertex is an element of $\mathbf{Y}$.

- The length of a path is the number of edges that the path uses, counting multiple edges multiple times.

- Let $\mathcal{V}_{1}$ and $\mathcal{V}_{2}$ denote two subsets of $\mathcal{V}$. The cardinality of $\mathcal{V}_{1}$ is noted card $\left(\mathcal{V}_{1}\right)$. A path $P$ is said a $\mathcal{V}_{1}-\mathcal{V}_{2}$ path if its begin vertex belongs to $\mathcal{V}_{1}$ and its end vertex belongs to $\mathcal{V}_{2}$. If the only vertices of $P$ belonging to $\mathcal{V}_{1} \cup \mathcal{V}_{2}$ are its begin and its end vertices, $P$ is said a direct $\mathcal{V}_{1}-\mathcal{V}_{2}$ path.

- A set of $l$ disjoint $\mathcal{V}_{1}-\mathcal{V}_{2}$ paths is called a $\mathcal{V}_{1}-\mathcal{V}_{2}$ linking of size $l$. The linkings which consist of a maximal number of disjoint $\mathcal{V}_{1}-\mathcal{V}_{2}$ paths are called maximum $\mathcal{V}_{1}-\mathcal{V}_{2}$ linkings. We define by $\rho\left(\mathcal{V}_{1}, \mathcal{V}_{2}\right)$ the size of these maximum $\mathcal{V}_{1}-\mathcal{V}_{2}$ linkings.

- The length of a $\mathcal{V}_{1}-\mathcal{V}_{2}$ linking is defined as the sum of the lengths of all its paths.

- $\mu\left(\mathcal{V}_{1}, \mathcal{V}_{2}\right)$ is the minimal number of vertices belonging to a maximum $\mathcal{V}_{1}-\mathcal{V}_{2}$ linking. Note that the minimal length of a maximum $\mathcal{V}_{1}-\mathcal{V}_{2}$ linking is equal to $\mu\left(\mathcal{V}_{1}, \mathcal{V}_{2}\right)-\rho\left(\mathcal{V}_{1}, \mathcal{V}_{2}\right)$.

- $V_{\text {ess }}\left(\mathcal{V}_{1}, \mathcal{V}_{2}\right) \stackrel{\text { def }}{=}\{\mathbf{v} \in \mathcal{V} \mid \mathbf{v}$ is included in every maximum $\mathcal{V}_{1}-\mathcal{V}_{2}$ linking $\}$. Vertex subset $V_{\text {ess }}\left(\mathcal{V}_{1}, \mathcal{V}_{2}\right)$ denotes the set of all essential vertices (van der Woude, 2000), which correspond by definition to vertices present in all the maximum $\mathcal{V}_{1}-\mathcal{V}_{2}$ linkings.

- $\mathbf{S} \subseteq \mathcal{V}$ is a separator between sets $\mathcal{V}_{1}$ and $\mathcal{V}_{2}$, if every path from $\mathcal{V}_{1}$ to $\mathcal{V}_{2}$ contains at least one vertex in $\mathrm{S}$. We call minimum separators between $\mathcal{V}_{1}$ and $\mathcal{V}_{2}$ any separators having the smallest size. According to Menger's Theorem, the latter equals $\rho\left(\mathcal{V}_{1}, \mathcal{V}_{2}\right)$.

- There exist two uniquely determined minimum separators between $\mathcal{V}_{1}$ and $\mathcal{V}_{2}$ noted $\mathbf{S}^{\mathbf{i}}\left(\mathcal{V}_{1}, \mathcal{V}_{2}\right)$ and $\mathbf{S}^{\mathbf{o}}\left(\mathcal{V}_{1}, \mathcal{V}_{2}\right)$ such that (van der Woude, 2000):

- $\mathbf{S}\left(\mathcal{V}_{1}, \mathcal{V}_{2}\right)$ is the set of the end vertices of all direct $\mathcal{V}_{1}-$ $V_{\text {ess }}\left(\mathcal{V}_{1}, \mathcal{V}_{2}\right)$ paths, where $V_{\text {ess }}\left(\mathcal{V}_{1}, \mathcal{V}_{2}\right) \cap \mathcal{V}_{1}$ is considered, in the present definition, as output vertices. Vertex subset $\mathbf{S}^{\mathbf{i}}\left(\mathcal{V}_{1}, \mathcal{V}_{2}\right)$ is the minimum input separator.

- $\mathbf{S}^{\mathbf{o}}\left(\mathcal{V}_{1}, \mathcal{V}_{2}\right)$ is the set of begin vertices of all direct $V_{\text {ess }}\left(\mathcal{V}_{1}, \mathcal{V}_{2}\right)-\mathcal{V}_{2}$ paths, where $V_{\text {ess }}\left(\mathcal{V}_{1}, \mathcal{V}_{2}\right) \cap \mathcal{V}_{2}$ is considered, in the present definition, as input vertices. Vertex subset $\mathbf{S}^{\mathbf{o}}\left(\mathcal{V}_{1}, \mathcal{V}_{2}\right)$ is the minimum output separator.
It results, from the previous definitions, that

$V_{\text {ess }}\left(\mathcal{V}_{1}, \mathcal{V}_{2}\right) \cap \mathcal{V}_{1} \subseteq \mathbf{S}^{\mathbf{i}}\left(\mathcal{V}_{1}, \mathcal{V}_{2}\right)$ and $V_{\text {ess }}\left(\mathcal{V}_{1}, \mathcal{V}_{2}\right) \cap \mathcal{V}_{2} \subseteq$ $\mathbf{S}^{\mathbf{o}}\left(\mathcal{V}_{1}, \mathcal{V}_{2}\right)$.

- $\theta\left(\mathcal{V}_{1}, \mathcal{V}_{2}\right)$ is the maximal number of $v$-disjoint edges from $\mathcal{V}_{1}$ to $\mathcal{V}_{2}$.

In Example 3, $\rho(\mathbf{U}, \mathbf{Y})=2$ and $\theta(\mathbf{U}, \mathbf{Y})=1$. Furthermore, there exist several maximum linkings:

$\left\{\mathrm{u}_{1} \rightarrow \mathrm{x}_{3} \rightarrow \mathrm{x}_{4} \rightarrow \mathrm{x}_{5} \rightarrow \mathrm{y}_{1} ; \mathrm{u}_{2} \rightarrow \mathrm{y}_{4}\right\}$

$\left\{\mathrm{u}_{1} \rightarrow \mathrm{x}_{3} \rightarrow \mathrm{x}_{4} \rightarrow \mathrm{x}_{6} \rightarrow \mathrm{x}_{5} \rightarrow \mathrm{y}_{1} ; \mathrm{u}_{2} \rightarrow \mathrm{x}_{8} \rightarrow \mathrm{y}_{4}\right\}, \ldots$ According to the fact that the first maximum linking is the "shortest one" with a length equal to 5 , we have that $\mu(\mathbf{U}, \mathbf{Y})=7$. Finally, $V_{\text {ess }}(\mathbf{U}, \mathbf{Y})=\left\{\mathbf{u}_{\mathbf{1}}, \mathbf{x}_{\mathbf{3}}, \mathbf{x}_{\mathbf{5}}, \mathbf{u}_{\mathbf{2}}\right\}$, $\mathbf{S}^{\mathbf{i}}(\mathbf{U}, \mathbf{Y})=\left\{\mathbf{u}_{1}, \mathbf{u}_{2}\right\}$ and $\mathbf{S}^{\mathbf{o}}(\mathbf{U}, \mathbf{Y})=\left\{\mathbf{x}_{\mathbf{5}}, \mathbf{u}_{\mathbf{2}}\right\}$.

\section{Main results}

\subsection{Preliminary results}

At first, let us specify a particular subdivision of structured system $\Sigma_{\Lambda}$ :

Definition 4 For structured system $\Sigma_{\Lambda}$ represented by digraph $\mathcal{G}\left(\Sigma_{\Lambda}\right)$, we define the vertex subsets:

$\boldsymbol{\Delta}_{\mathbf{0}} \stackrel{\text { def }}{=}\left\{\mathbf{x}_{\mathbf{i}} \mid \rho\left(\mathbf{U} \cup\left\{\mathbf{x}_{\mathbf{i}}\right\}, \mathbf{Y}\right)=\rho(\mathbf{U}, \mathbf{Y})\right\} ;$

$\mathbf{X}_{\mathbf{1}} \stackrel{\text { def }}{=}\left\{\mathbf{x}_{\mathbf{i}} \mid \rho\left(\mathbf{U} \cup\left\{\mathbf{x}_{\mathbf{i}}\right\}, \mathbf{Y}\right)>\rho(\mathbf{U}, \mathbf{Y})\right\}=\mathbf{X} \backslash \boldsymbol{\Delta}_{\mathbf{0}}$;

$\mathbf{Y}_{\mathbf{0}} \stackrel{\text { def }}{=}\left\{\mathbf{y}_{\mathbf{i}} \mid \rho(\mathbf{U}, \mathbf{Y})>\rho\left(\mathbf{U}, \mathbf{Y} \backslash\left\{\mathbf{y}_{\mathbf{i}}\right\}\right)\right\}=\mathbf{Y} \cap V_{\text {ess }}(\mathbf{U}, \mathbf{Y})$;

$\mathbf{Y}_{\mathbf{1}} \stackrel{\text { def }}{=} \mathbf{Y} \backslash \mathbf{Y}_{\mathbf{0}} ; \mathbf{U}_{\mathbf{0}} \stackrel{\text { def }}{=}\left\{\mathbf{u}_{\mathbf{i}} \mid \theta\left(\left\{\mathbf{u}_{\mathbf{i}}\right\}, \mathbf{X}_{\mathbf{1}} \cup \mathbf{Y}_{\mathbf{1}}\right)=0\right\}$;

$\mathbf{U}_{\mathbf{1}} \stackrel{\text { def }}{=} \mathbf{U} \backslash \mathbf{U}_{\mathbf{0}} ; \mathbf{X}_{\mathbf{s}} \stackrel{\text { def }}{=} \mathbf{S}^{\mathbf{o}}\left(\mathbf{U}_{\mathbf{0}}, \mathbf{Y}\right) \cap \mathbf{X}$ and $\mathbf{X}_{\mathbf{0}} \stackrel{\text { def }}{=} \boldsymbol{\Delta}_{\mathbf{0}} \backslash \mathbf{X}_{\mathbf{s}}$. Furthermore, we define $n_{0}=\operatorname{card}\left(\mathbf{X}_{\mathbf{0}}\right), n_{s}=\operatorname{card}\left(\mathbf{X}_{\mathbf{s}}\right)$, $n_{1}=\operatorname{card}\left(\mathbf{X}_{\mathbf{1}}\right), q_{0}=\operatorname{card}\left(\mathbf{U}_{\mathbf{0}}\right), q_{1}=\operatorname{card}\left(\mathbf{U}_{\mathbf{1}}\right)$, $p_{0}=\operatorname{card}\left(\mathbf{Y}_{\mathbf{0}}\right)$ and $p_{1}=\operatorname{card}\left(\mathbf{Y}_{\mathbf{1}}\right)$.

Considering the system described in Example 3, we have mentioned that $\rho(\mathbf{U}, \mathbf{Y})=2$. Since $\mathbf{Y} \cap V_{\text {ess }}(\mathbf{U}, \mathbf{Y})=\emptyset$, we have that $\mathbf{Y}_{\mathbf{0}}=\emptyset, \mathbf{Y}_{\mathbf{1}}=\mathbf{Y}$. Moreover, for $i=1, \ldots, 9$, let us compute the number of disjoint paths from $\mathbf{U} \cup\left\{\mathbf{x}_{\mathbf{i}}\right\}$ to $\mathbf{Y}$ :

for $i=1, \ldots, 6, \rho\left(\mathbf{U} \cup\left\{\mathbf{x}_{\mathbf{i}}\right\}, \mathbf{Y}\right)=2$ and for $i=$ $7, \ldots, 9, \rho\left(\mathbf{U} \cup\left\{\mathbf{x}_{\mathbf{i}}\right\}, \mathbf{Y}\right)=3$. We can deduce that $\boldsymbol{\Delta}_{0}=\left\{\mathbf{x}_{1}, \mathbf{x}_{2}, \mathbf{x}_{3}, \mathbf{x}_{4}, \mathbf{x}_{5}, \mathbf{x}_{6}\right\}, \mathbf{X}_{1}=\left\{\mathbf{x}_{7}, \mathbf{x}_{8}, \mathbf{x}_{9}\right\}$. Furthermore, contrary to $\mathbf{u}_{2}, \mathbf{u}_{1}$ cannot be linked with an edge to an element of $\mathbf{X}_{1} \cup \mathbf{Y}_{1}$, so $\mathbf{U}_{0}=\left\{\mathbf{u}_{1}\right\}$ and $\mathbf{U}_{\mathbf{1}}=\left\{\mathbf{u}_{\mathbf{2}}\right\}$. Finally, $V_{\text {ess }}\left(\mathbf{U}_{\mathbf{0}}, \mathbf{Y}\right)=\left\{\mathbf{u}_{\mathbf{1}}, \mathbf{x}_{\mathbf{3}}, \mathbf{x}_{\mathbf{5}}\right\}$ and so $\mathbf{S}^{\mathbf{o}}\left(\mathbf{U}_{\mathbf{0}}, \mathbf{Y}\right)=\left\{\mathbf{x}_{\mathbf{5}}\right\}$. Thus, $\mathbf{X}_{\mathbf{s}}=\left\{\mathbf{x}_{\mathbf{5}}\right\}$ and $\mathbf{X}_{0}=\Delta_{0} \backslash \mathbf{X}_{\mathrm{s}}=\left\{\mathbf{x}_{1}, \mathbf{x}_{2}, \mathbf{x}_{3}, \mathbf{x}_{4}, \mathbf{x}_{6}\right\}$.

Let us comment the graph partition presented above:

- $\boldsymbol{\Delta}_{\mathbf{0}}$ merges the state vertices which cannot be linked to $\mathbf{Y}$, the state vertices belonging to $V_{\text {ess }}(\mathbf{U}, \mathbf{Y})$. Obviously, $V_{e s s}(\mathbf{U}, \mathbf{Y}) \cap \mathbf{X} \subseteq \boldsymbol{\Delta}_{\mathbf{0}}$ and the state vertices from which all $\mathbf{Y}$-topped paths lead to $V_{\text {ess }}(\mathbf{U}, \mathbf{Y})$.

Indeed, if it is not the case i.e. there exist $\mathbf{v}_{\mathbf{i}} \in \boldsymbol{\Delta}_{\mathbf{0}}$ and a path $P$ from $\mathbf{v}_{\mathbf{i}}$ to $\mathbf{Y}$ which does not contain any element of $V_{\text {ess }}(\mathbf{U}, \mathbf{Y})$, then there is no element of $\mathbf{S}^{\mathbf{o}}(\mathbf{U}, \mathbf{Y})$ in $P$ and so $\operatorname{card}\left(\mathbf{S}^{\mathbf{o}}\left(\mathbf{U} \cup\left\{\mathbf{v}_{\mathbf{i}}\right\}, \mathbf{Y}\right)\right)>$ $\operatorname{card}\left(\mathbf{S}^{\mathbf{o}}(\mathbf{U}, \mathbf{Y})\right)$. According to Menger's Theorem, this implies that $\rho\left(\mathbf{U} \cup\left\{\mathbf{v}_{\mathbf{i}}\right\}, \mathbf{Y}\right)>\rho(\mathbf{U}, \mathbf{Y})$, which is in contradiction with assumption $\mathbf{v}_{\mathbf{i}} \in \boldsymbol{\Delta}_{\mathbf{0}}$.

- Directly from the definition of $\mathbf{Y}_{\mathbf{0}}$, we have that $\mathbf{Y}_{\mathbf{0}} \subseteq$ $V_{e s s}(\mathbf{U}, \mathbf{Y})$ and so $\mathbf{Y}_{\mathbf{0}} \subseteq \mathbf{S}^{\mathbf{o}}(\mathbf{U}, \mathbf{Y})$. 
- Assume that $\rho(\mathbf{U}, \mathbf{Y})=q$, then $\rho\left(\mathbf{U}_{\mathbf{1}}, \mathbf{Y}\right)=\operatorname{card}\left(\mathbf{U}_{\mathbf{1}}\right)$. In this case, $\mathbf{U}_{\mathbf{1}} \subseteq V_{e s s}(\mathbf{U}, \mathbf{Y})$ and subsequently $\mathbf{U}_{\mathbf{1}} \subseteq$ $\mathbf{S}^{\mathrm{i}}(\mathbf{U}, \mathbf{Y})$. Moreover, all elements of $\mathbf{U}_{1}$ are begin vertices of $\mathbf{U}-\mathbf{Y}$ paths where all vertices are in $\mathbf{U}_{\mathbf{1}} \cup \mathbf{X}_{\mathbf{1}} \cup \mathbf{Y}_{\mathbf{1}}$. Since, $\left(\mathbf{X}_{\mathbf{1}} \cup \mathbf{Y}_{\mathbf{1}}\right) \cap V_{\text {ess }}(\mathbf{U}, \mathbf{Y})=\emptyset$, all elements of $\mathbf{U}_{\mathbf{1}}$ are begin vertices of direct $V_{\text {ess }}(\mathbf{U}, \mathbf{Y})-\mathbf{Y}$ paths and so $\mathbf{U}_{\mathbf{1}} \subseteq \mathbf{S}^{\mathbf{o}}(\mathbf{U}, \mathbf{Y})$. Otherwise, $\mathbf{U}_{\mathbf{1}} \subseteq \mathbf{S}^{\mathbf{i}}(\mathbf{U}, \mathbf{Y})$ and $\mathbf{U}_{\mathbf{1}} \subseteq \mathbf{S}^{\mathbf{o}}(\mathbf{U}, \mathbf{Y})$ imply that, in a maximum $\mathbf{U}-\mathbf{Y}$ linking, all vertices included in a path starting from $\mathbf{U}_{1}$ are not included in $V_{e s s}(\mathbf{U}, \mathbf{Y})$ and so are not included in $\boldsymbol{\Delta}_{\mathbf{0}}$. So,

$$
V_{e s s}(\mathbf{U}, \mathbf{Y})=\mathbf{U}_{\mathbf{1}} \cup V_{\text {ess }}\left(\mathbf{U}_{\mathbf{0}}, \mathbf{Y}\right)
$$

- From each $\mathbf{x}_{\mathbf{i}} \in \mathbf{X}_{\mathbf{1}}$, there exists an $\mathbf{Y}$-topped path, which is disjoint with at least one maximum $\mathbf{U}-\mathbf{Y}$ linking. Therefore, $\forall \mathbf{x}_{\mathbf{i}} \in \mathbf{X}_{\mathbf{1}}$, there exists an $\mathbf{Y}$-topped path constituted only by the elements of $\mathbf{X}_{1}$ and $\mathbf{Y}_{\mathbf{1}}$. In the same sense, $\forall \mathbf{x}_{\mathbf{i}} \in \mathbf{X}_{\mathbf{1}}$, in all the maximum $\mathbf{U} \cup\left\{\mathbf{x}_{\mathbf{i}}\right\}-\mathbf{Y}$ linkings, the paths beginning with the vertices of $\mathbf{U}_{\mathbf{1}} \cup\left\{\mathbf{x}_{\mathbf{i}}\right\}$ are necessarily in $\mathbf{X}_{\mathbf{1}} \cup \mathbf{Y}_{\mathbf{1}}$. Similarly, $\forall \mathbf{y}_{\mathbf{i}} \in \mathbf{Y}_{\mathbf{1}}$, in all the maximum $\mathbf{U}-\mathbf{Y} \backslash\left\{\mathbf{y}_{\mathbf{i}}\right\}$ linkings, the paths beginning with the vertices of $U_{1}$ are necessarily in $X_{1} \cup Y_{1}$. Thus, $\forall \mathbf{x}_{\mathbf{i}} \in \mathbf{X}_{\mathbf{1}}$ and $\forall \mathbf{y}_{\mathbf{i}} \in \mathbf{Y}_{\mathbf{1}}$,

$$
\begin{aligned}
& V_{e s s}\left(\mathbf{U} \cup\left\{\mathbf{x}_{\mathbf{i}}\right\}, \mathbf{Y}\right) \cap\left(\boldsymbol{\Delta}_{\mathbf{0}} \cup \mathbf{Y}_{\mathbf{0}}\right)= \\
& V_{\text {ess }}\left(\mathbf{U}, \mathbf{Y} \backslash\left\{\mathbf{y}_{\mathbf{i}}\right\}\right) \cap\left(\boldsymbol{\Delta}_{\mathbf{0}} \cup \mathbf{Y}_{\mathbf{0}}\right)=V_{\text {ess }}\left(\mathbf{U}_{\mathbf{0}}, \mathbf{Y}\right)
\end{aligned}
$$

According to Definition 4, we state now:

Lemma 5 Consider structured system $\Sigma_{\Lambda}$ represented by digraph $\mathcal{G}\left(\Sigma_{\Lambda}\right)$, where $\rho(\mathbf{U}, \mathbf{Y})=q$. Using the subdivision given in Definition 4, we have:

St1. $\mathbf{S}^{\mathbf{o}}\left(\mathbf{U}_{\mathbf{0}}, \mathbf{Y}\right) \cap\left(\mathbf{X}_{1} \cup \mathbf{Y}_{\mathbf{1}}\right)=\emptyset$;

St2. $\forall \mathrm{v}_{\mathrm{i}} \in \Delta_{0} \cup \mathrm{U}_{0}$,

$\mathbf{v}_{\mathbf{i}} \in \mathbf{S}^{\mathbf{o}}\left(\mathbf{U}_{\mathbf{0}}, \mathbf{Y}\right) \Leftrightarrow \theta\left(\left\{\mathbf{v}_{\mathbf{i}}\right\}, \mathbf{X}_{\mathbf{1}} \cup \mathbf{Y}_{\mathbf{1}}\right) \neq 0 ;$

St3. $\theta\left(\mathbf{X}_{\mathbf{s}}, \mathbf{X}_{\mathbf{1}} \cup \mathbf{Y}_{\mathbf{1}}\right)=\operatorname{card}\left(\mathbf{X}_{\mathbf{s}}\right)=n_{s}$;

St4. $\mathbf{S}^{\mathbf{o}}\left(\mathbf{U}_{0}, \mathbf{Y}\right) \cap \mathbf{U}_{0}=\emptyset$;

St5. $\theta\left(\mathbf{X}_{\mathbf{0}}, \mathbf{X}_{\mathbf{1}} \cup \mathbf{Y}_{\mathbf{1}}\right)=0$;

St6. $\mathbf{Y}_{\mathbf{0}} \subseteq \mathbf{S}^{\mathbf{o}}\left(\mathbf{U}_{\mathbf{0}}, \mathbf{Y}\right)$.

\section{Proof:}

[St1.] Due to the definition of $\mathbf{X}_{1}$ and $\mathbf{Y}_{1},\left(\mathbf{X}_{1} \cup \mathbf{Y}_{\mathbf{1}}\right) \cap$ $V_{e s s}(\mathbf{U}, \mathbf{Y})=\emptyset$. Yet, $\mathbf{S}^{\circ}\left(\mathbf{U}_{\mathbf{0}}, \mathbf{Y}\right) \subseteq V_{e s s}\left(\mathbf{U}_{\mathbf{0}}, \mathbf{Y}\right) \subseteq$ $V_{\text {ess }}(\mathbf{U}, \mathbf{Y})$. Then, $\mathbf{S}^{\mathbf{o}}\left(\mathbf{U}_{\mathbf{0}}, \mathbf{Y}\right) \cap\left(\mathbf{X}_{\mathbf{1}} \cup \mathbf{Y}_{\mathbf{1}}\right)=\emptyset$.

[St2.] First, we show that $\forall \mathbf{v}_{\mathbf{i}} \in \boldsymbol{\Delta}_{\mathbf{0}}$, if $\theta\left(\left\{\mathbf{v}_{\mathbf{i}}\right\}, \mathbf{X}_{\mathbf{1}} \cup \mathbf{Y}_{\mathbf{1}}\right) \neq$ 0 then $\mathbf{v}_{\mathbf{i}} \in \mathbf{S}^{\mathbf{o}}(\mathbf{U}, \mathbf{Y})$.

We have that $\mathbf{v}_{\mathbf{i}} \in \boldsymbol{\Delta}_{\mathbf{0}}$ and $\theta\left(\left\{\mathbf{v}_{\mathbf{i}}\right\}, \mathbf{X}_{\mathbf{1}} \cup \mathbf{Y}_{\mathbf{1}}\right) \neq 0$ imply that $\mathbf{v}_{\mathbf{i}} \in V_{\text {ess }}(\mathbf{U}, \mathbf{Y})$. Indeed, if is assumed that it is not the case, then according to relations (4), $\forall \mathbf{x}_{\mathbf{j}} \in \mathbf{X}_{\mathbf{1}}, \mathbf{v}_{\mathbf{i}} \notin V_{e s s}\left(\mathbf{U} \cup\left\{\mathbf{x}_{\mathbf{j}}\right\}, \mathbf{Y}\right)$ and $\forall \mathbf{y}_{\mathbf{k}} \in \mathbf{Y}_{\mathbf{1}}$, $\mathbf{v}_{\mathbf{i}} \notin V_{\text {ess }}\left(\mathbf{U}, \mathbf{Y} \backslash\left\{\mathbf{y}_{\mathbf{k}}\right\}\right)$. Since $\mathbf{v}_{\mathbf{i}}$ is directly linked to $\mathbf{X}_{\mathbf{1}} \cup \mathbf{Y}_{\mathbf{1}}$, then there exists a $\left\{\mathbf{v}_{\mathbf{i}}\right\}-\mathbf{Y}$ path which is disjoint from all the paths constituting a maximum $\mathbf{U}-\mathbf{Y}$ linking. This is equivalent to $\rho\left(\mathbf{U} \cup\left\{\mathbf{v}_{\mathbf{i}}\right\}, \mathbf{Y}\right)>\rho(\mathbf{U}, \mathbf{Y})$, which is in contradiction with the fact that $\mathbf{v}_{\mathbf{i}} \in \Delta_{0}$. Furthermore, not only $\mathbf{v}_{\mathbf{i}} \in V_{\text {ess }}(\mathbf{U}, \mathbf{Y})$ but also it is the begin vertex of a direct $V_{e s s}(\mathbf{U}, \mathbf{Y})-\mathbf{Y}$ path. Thus, $\mathbf{v}_{\mathbf{i}} \in \mathbf{S}^{\mathbf{o}}(\mathbf{U}, \mathbf{Y})$.

Moreover, from equality (3), $\mathbf{v}_{\mathbf{i}} \in V_{\text {ess }}\left(\mathbf{U}_{\mathbf{0}}, \mathbf{Y}\right)$ and as $\mathbf{v}_{\mathbf{i}}$ is the begin vertex of a direct $V_{\text {ess }}\left(\mathbf{U}_{\mathbf{0}}, \mathbf{Y}\right)-\mathbf{Y}$ path, this implies that $\mathbf{v}_{\mathbf{i}} \in \mathbf{S}^{\mathbf{o}}\left(\mathbf{U}_{\mathbf{0}}, \mathbf{Y}\right)$.

Now, we will prove that $\forall \mathbf{v}_{\mathbf{i}} \in \boldsymbol{\Delta}_{\mathbf{0}} \cup \mathbf{U}_{\mathbf{0}}, \theta\left(\left\{\mathbf{v}_{\mathbf{i}}\right\}, \mathbf{X}_{\mathbf{1}} \cup\right.$
$\left.\mathbf{Y}_{\mathbf{1}}\right)=0 \Rightarrow \mathbf{v}_{\mathbf{i}} \notin \mathbf{S}^{\mathbf{o}}\left(\mathbf{U}_{\mathbf{0}}, \mathbf{Y}\right)$.

Assume that there exists $\mathbf{v}_{\mathbf{i}} \in \boldsymbol{\Delta}_{\mathbf{0}} \cup \mathbf{U}_{\mathbf{0}}$ such that $\theta\left(\left\{\mathbf{v}_{\mathbf{i}}\right\}, \mathbf{X}_{\mathbf{1}} \cup \mathbf{Y}_{\mathbf{1}}\right)=0$ and $\mathbf{v}_{\mathbf{i}} \in \mathbf{S}^{\mathbf{o}}\left(\mathbf{U}_{\mathbf{0}}, \mathbf{Y}\right)$. Obviously, $\mathbf{v}_{\mathbf{i}} \in \mathbf{S}^{\mathbf{o}}\left(\mathbf{U}_{\mathbf{0}}, \mathbf{Y}\right)$ implies that $\mathbf{v}_{\mathbf{i}}$ is included in all maximum $\mathbf{U}_{\mathbf{0}}-\mathbf{Y}$ linkings. Consider any maximum $\mathbf{U}_{0}-\mathbf{Y}$ linking, it necessarily includes a path of the form $P=\mathbf{u}_{\mathbf{j}} \rightarrow \ldots \rightarrow \mathbf{v}_{\mathbf{i}} \rightarrow \mathbf{v}_{\mathbf{j}_{1}} \rightarrow \mathbf{v}_{\mathbf{j}_{2}} \ldots \rightarrow \mathbf{v}_{\mathbf{j}_{\mathbf{k}}} \rightarrow \mathbf{y}_{\mathbf{t}}$. First note that as $\theta\left(\left\{\mathbf{v}_{\mathbf{i}}\right\}, \mathbf{X}_{\mathbf{1}} \cup \mathbf{Y}_{\mathbf{1}}\right)=0, \mathbf{v}_{\mathbf{j}_{\mathbf{1}}} \in \boldsymbol{\Delta}_{\mathbf{0}}$. Next, for $r>1$ if $\mathbf{v}_{\mathbf{j}_{\mathbf{r}}} \in \mathbf{X}_{\mathbf{1}}$ then from the previous settings $\mathbf{v}_{\mathbf{j}_{\mathbf{r}-1}} \in \mathbf{S}^{\mathbf{o}}\left(\mathbf{U}_{\mathbf{0}}, \mathbf{Y}\right)$. Nevertheless, this is impossible because in any maximum $\mathbf{U}_{0^{-}} \mathbf{Y}$ linkings, we must have one and only one element of $\mathbf{S}^{\mathbf{o}}\left(\mathbf{U}_{\mathbf{0}}, \mathbf{Y}\right)$ in every path. So, $\mathbf{v}_{\mathbf{j}_{\mathbf{1}}}, \mathbf{v}_{\mathbf{j}_{\mathbf{2}}} \ldots, \mathbf{v}_{\mathbf{j}_{\mathbf{k}}}$ are all included in $\boldsymbol{\Delta}_{\mathbf{0}}$. Besides, $\mathbf{y}_{\mathbf{t}} \notin \mathbf{Y}_{\mathbf{1}}$ because otherwise $\mathbf{v}_{\mathbf{j}_{\mathbf{k}}} \in \mathbf{S}^{\mathbf{o}}\left(\mathbf{U}_{\mathbf{0}}, \mathbf{Y}\right)$. This implies necessarily that, $\mathbf{y}_{\mathbf{t}} \in \mathbf{Y}_{\mathbf{0}}$. By definition, $\mathbf{Y}_{\mathbf{0}} \subseteq V_{\text {ess }}(\mathbf{U}, \mathbf{Y})$ and from equality (3), we have $\mathbf{y}_{\mathbf{t}} \in V_{\text {ess }}\left(\mathbf{U}_{\mathbf{0}}, \mathbf{Y}\right)$. Thus, $\mathbf{y}_{\mathbf{t}} \in \mathbf{S}^{\mathbf{o}}\left(\mathbf{U}_{\mathbf{0}}, \mathbf{Y}\right)$. Hence, in path $P$, we have two elements of $\mathbf{S}^{\mathbf{O}}\left(\mathbf{U}_{\mathbf{0}}, \mathbf{Y}\right)$. As this fact is impossible, the assumption that there exists $\mathbf{v}_{\mathbf{i}} \in \boldsymbol{\Delta}_{\mathbf{0}} \cup \mathbf{U}_{\mathbf{0}}$ such that $\theta\left(\left\{\mathbf{v}_{\mathbf{i}}\right\}, \mathbf{X}_{\mathbf{1}} \cup \mathbf{Y}_{\mathbf{1}}\right)=0$ and $\mathbf{v}_{\mathbf{i}} \in \mathbf{S}^{\mathbf{o}}\left(\mathbf{U}_{\mathbf{0}}, \mathbf{Y}\right)$ is false and [St2] is proved.

[St3.] In any maximum $\mathbf{U}_{0^{-}} \mathbf{Y}$ linking, there are $n_{s}$ paths containing vertices of $\mathbf{X}_{\mathbf{s}}$ which end necessarily with a vertex of $\mathbf{Y}_{1}$. Moreover, in all these paths, all state vertices between elements of $\mathbf{X}_{\mathbf{s}}$ and output vertices belong to $\mathbf{X}_{\mathbf{1}}$. Hence, there exist $n_{s}$ disjoint paths linking $\mathbf{X}_{\mathbf{s}}$ to $\mathbf{Y}_{\mathbf{1}}$ and including only vertices of $\mathbf{X}_{\mathbf{1}} \cup \mathbf{Y}_{\mathbf{1}}$. So, $\theta\left(\mathbf{X}_{\mathbf{s}}, \mathbf{X}_{\mathbf{1}} \cup \mathbf{Y}_{\mathbf{1}}\right)=n_{s}$.

[St4.] As $\theta\left(\mathbf{U}_{\mathbf{0}}, \mathbf{X}_{\mathbf{1}} \cup \mathbf{Y}_{\mathbf{1}}\right)=0$, we have from [St2] that $\mathbf{U}_{\mathbf{0}} \cap \mathbf{S}^{\mathbf{o}}\left(\mathbf{U}_{\mathbf{0}}, \mathbf{Y}\right)=\emptyset$.

[St5.] Since $\mathbf{X}_{\mathbf{0}}=\mathbf{X} \backslash\left(\mathbf{X}_{\mathrm{s}} \cup \mathbf{X}_{\mathbf{1}}\right)$, where $\mathbf{X}_{\mathrm{s}}=$ $\mathbf{S}^{\mathbf{o}}\left(\mathbf{U}_{\mathbf{0}}, \mathbf{Y}\right) \cap \mathbf{X}$, we have that $\mathbf{S}^{\mathbf{o}}\left(\mathbf{U}_{\mathbf{0}}, \mathbf{Y}\right) \cap \mathbf{X}_{\mathbf{0}}=\emptyset$. So, directly from [St2], $\theta\left(\mathbf{X}_{\mathbf{0}}, \mathbf{X}_{\mathbf{1}} \cup \mathbf{Y}_{\mathbf{1}}\right)=0$.

[St6.] From statement [St2], $\forall \mathbf{v}_{\mathbf{i}} \in \mathbf{S}^{\mathbf{o}}\left(\mathbf{U}_{\mathbf{0}}, \mathbf{Y}\right)$, satisfying $\theta\left(\left\{\mathbf{v}_{\mathbf{i}}\right\}, \mathbf{X}_{\mathbf{1}} \cup \mathbf{Y}_{\mathbf{1}}\right) \neq 0$ we have that $\mathbf{v}_{\mathbf{i}} \in \mathbf{S}^{\mathbf{o}}(\mathbf{U}, \mathbf{Y})$ and so that $\mathbf{S}^{\mathbf{o}}\left(\mathbf{U}_{\mathbf{0}}, \mathbf{Y}\right) \subseteq \mathbf{S}^{\mathbf{o}}(\mathbf{U}, \mathbf{Y})$. Besides, $\mathbf{U}_{\mathbf{1}} \subseteq \mathbf{S}^{\mathbf{o}}(\mathbf{U}, \mathbf{Y})$, $\mathbf{U}_{\mathbf{1}} \cap \mathbf{S}^{\mathbf{o}}\left(\mathbf{U}_{\mathbf{0}}, \mathbf{Y}\right)=\emptyset$ and $\operatorname{card}\left(\mathbf{S}^{\mathbf{o}}(\mathbf{U}, \mathbf{Y})\right)=q_{1}+q_{0}=$ $\operatorname{card}\left(\mathbf{U}_{\mathbf{1}}\right)+\operatorname{card}\left(\mathbf{S}^{\mathbf{o}}\left(\mathbf{U}_{\mathbf{0}}, \mathbf{Y}\right)\right)$. Thus, $\mathbf{S}^{\mathbf{o}}(\mathbf{U}, \mathbf{Y})=$ $\mathbf{S}^{\mathbf{0}}\left(\mathbf{U}_{\mathbf{0}}, \mathbf{Y}\right) \cup \mathbf{U}_{\mathbf{1}}$. Yet, $\mathbf{Y}_{\mathbf{0}} \subseteq V_{\text {ess }}(\mathbf{U}, \mathbf{Y}) \Rightarrow \mathbf{Y}_{\mathbf{0}} \subseteq$ $\mathbf{S}^{\mathbf{o}}(\mathbf{U}, \mathbf{Y})$ and as $\mathbf{S}^{\mathbf{o}}(\mathbf{U}, \mathbf{Y})=\mathbf{S}^{\mathbf{o}}\left(\mathbf{U}_{\mathbf{0}}, \mathbf{Y}\right) \cup \mathbf{U}_{\mathbf{1}}$, we have finally $\mathbf{Y}_{\mathbf{0}} \subseteq \mathbf{S}^{\mathbf{o}}\left(\mathbf{U}_{\mathbf{0}}, \mathbf{Y}\right)$.

To summarize, Lemma 6 states that

$V_{e s s}(\mathbf{U}, \mathbf{Y})=V_{\text {ess }}\left(\mathbf{U}_{\mathbf{0}}, \mathbf{Y}\right) \cup \mathbf{U}_{\mathbf{1}}, \theta\left(\mathbf{X}_{\mathbf{s}}, \mathbf{X}_{\mathbf{1}} \cup \mathbf{Y}_{\mathbf{1}}\right)=n_{s}$, $\mathbf{S}^{\mathbf{o}}(\mathbf{U}, \mathbf{Y})=\mathbf{S}^{\mathbf{o}}\left(\mathbf{U}_{\mathbf{0}}, \mathbf{Y}\right) \cup \mathbf{U}_{\mathbf{1}}=\mathbf{X}_{\mathbf{s}} \cup \mathbf{Y}_{\mathbf{0}} \cup \mathbf{U}_{\mathbf{1}}$ and that $\theta\left(\mathbf{X}_{\mathbf{0}} \cup \mathbf{Y}_{\mathbf{0}}, \mathbf{X}_{\mathbf{1}} \cup \mathbf{Y}_{\mathbf{1}}\right)=0$.

These equalities are important in the sequel of the paper.

\subsection{Generic input and state observability}

Using the graph decomposition presented above, we give now the first result of the paper

Proposition $6 \Sigma_{\Lambda}$ is generically state and input observable iff, in its associated digraph $\mathcal{G}\left(\Sigma_{\Lambda}\right)$, there exists a v-disjoint subgraph $\mathcal{S}_{\mathcal{G}}$ which satisfies:

Cond1. $\mathcal{S}_{\mathcal{G}}$ covers $\mathbf{X} \cup \mathbf{U}$;

Cond2. all vertices $\mathbf{x}_{\mathbf{i}}$ covered by cycles in $\mathcal{S}_{\mathcal{G}}$ are included in $\mathbf{X}_{1}$;

Cond3. $\mathcal{S}_{\mathcal{G}}$ contains a maximum $\mathbf{U}_{\mathbf{0}}-\mathbf{S}^{\mathbf{o}}\left(\mathbf{U}_{\mathbf{0}}, \mathbf{Y}\right)$ linking, with a length equal to $\mu\left(\mathbf{U}_{\mathbf{0}}, \mathbf{S}^{\circ}\left(\mathbf{U}_{\mathbf{0}}, \mathbf{Y}\right)\right)-\operatorname{card}\left(\mathbf{S}^{\circ}\left(\mathbf{U}_{\mathbf{0}}, \mathbf{Y}\right)\right)$. 
Proof: We first prove the necessity of Cond1, next we decompose the system into two subsystems and we study separately each of them.

Necessity of condition Cond1: Cond1 is equivalent to the existence of $n+q v$-disjoint edges and means that $g \_r a n k(P(0))=n+q$. Indeed, the number of $v$-disjoint edges in $\mathcal{G}\left(\Sigma_{\Lambda}\right)$ is equal to the maximum matching in the bipartite graph (Murota, 1987) associated to matrix $P(0)$, which is equal to $g \_r a n k(P(0))$. Therefore, Cond1 is a necessary condition to the generic input and state observability of $\Sigma_{\Lambda}$. Moreover, Cond1 implies also that $\rho(\mathbf{U}, \mathbf{Y})=q$ and so we can apply the results of Lemma 5.

Decomposition of system $\Sigma_{\Lambda}$ : using Definition 4 and results of Lemma 5, there is no edge from $\mathbf{X}_{\mathbf{0}} \cup \mathbf{U}_{\mathbf{0}}$ to $\mathbf{X}_{\mathbf{1}} \cup \mathbf{Y}_{\mathbf{1}}$. So, we can write $\Sigma_{\Lambda}$ as:

$$
\left\{\begin{aligned}
\dot{X}_{0}(t)= & A_{0,0}^{\lambda} X_{0}(t)+A_{0, s}^{\lambda} X_{s}(t)+A_{0,1}^{\lambda} X_{1}(t)+ \\
& +B_{0,0}^{\lambda} U_{0}(t)+B_{0,1}^{\lambda} U_{1}(t) \\
\dot{X}_{s}(t)= & A_{s, 0}^{\lambda} X_{0}(t)+A_{s, s}^{\lambda} X_{s}(t)+A_{s, 1}^{\lambda} X_{1}(t)+ \\
& +B_{s, 0}^{\lambda} U_{0}(t)+B_{s, 1}^{\lambda} U_{1}(t) \\
\dot{X}_{1}(t)= & A_{1, s}^{\lambda} X_{s}(t)+A_{1,1}^{\lambda} X_{1}(t)+B_{1,1}^{\lambda} U_{1}(t) \\
Y_{0}(t)= & C_{0,0}^{\lambda} X_{0}(t)+C_{0, s}^{\lambda} X_{s}(t)+C_{0,1}^{\lambda} X_{1}(t)+ \\
& +D_{0,0}^{\lambda} U_{0}(t)+D_{0,1}^{\lambda} U_{1}(t) \\
Y_{1}(t)= & C_{1, s}^{\lambda} X_{s}(t)+C_{1,1}^{\lambda} X_{1}(t)+D_{1,1}^{\lambda} U_{1}(t)
\end{aligned}\right.
$$

where $X_{0}, X_{s}, U_{0}, U_{1}, Y_{0}$ and $Y_{1}$ represent the variables associated to vertex subsets $\mathbf{X}_{\mathbf{0}}, \mathbf{X}_{\mathbf{s}}, \mathbf{U}_{\mathbf{0}}, \mathbf{U}_{\mathbf{1}}, \mathbf{Y}_{\mathbf{0}}$ and $\mathbf{Y}_{\mathbf{1}}$ respectively.

Therefore, with some appropriate permutations on the rows and columns of $P(s)$, we can transform $P(s)$ into

$$
\tilde{P}(s)=\left(\begin{array}{ccccc}
A_{0,0}^{\lambda}-s I_{n_{0}} & A_{0, s}^{\lambda} & B_{0,0}^{\lambda} & A_{0,1}^{\lambda} & B_{0,1}^{\lambda} \\
A_{s, 0}^{\lambda} & A_{s, s}^{\lambda}-s I_{n_{s}} & B_{s, 0}^{\lambda} & A_{s, 1}^{\lambda} & B_{s, 1}^{\lambda} \\
C_{0,0}^{\lambda} & C_{0, s}^{\lambda} & D_{0,0}^{\lambda} & C_{0,1}^{\lambda} & D_{0,1}^{\lambda} \\
0 & A_{1, s}^{\lambda} & 0 & A_{1,1}^{\lambda}-s I_{n_{1}} & B_{1,1}^{\lambda} \\
0 & C_{1, s}^{\lambda} & 0 & C_{1,1}^{\lambda} & D_{1,1}^{\lambda}
\end{array}\right) .
$$

According to statement St3, g_rank $\left(\begin{array}{c}A_{1, s}^{\lambda} \\ C_{1, s}^{\lambda}\end{array}\right)=\theta\left(\mathbf{X}_{\mathbf{s}}, \mathbf{X}_{\mathbf{1}} \cup\right.$

$\left.\mathbf{Y}_{\mathbf{1}}\right)=n_{s}$. Matrix $P(s)$, and so $\tilde{P}(s)$, has generically full column rank $\forall s \in \mathbb{C}$, iff $P_{e}(s)$ has also generically full column rank $\forall s \in \mathbb{C}$, where:

$$
P_{e}(s)=\left(\begin{array}{cccccc}
A_{0,0}^{\lambda}-s I_{n_{0}} & A_{0, s}^{\lambda} & B_{0,0}^{\lambda} & A_{0,1}^{\lambda} & B_{0,1}^{\lambda} & 0 \\
A_{s, 0}^{\lambda} & A_{s, s}^{\lambda}-s I_{n_{s}} & B_{s, 0}^{\lambda} & A_{s, 1}^{\lambda} & B_{s, 1}^{\lambda} & 0 \\
C_{0,0}^{\lambda} & C_{0, s}^{\lambda} & D_{0,0}^{\lambda} & C_{0,1}^{\lambda} & D_{0,1}^{\lambda} & 0 \\
0 & I_{n_{s}} & 0 & 0 & 0 & 0 \\
0 & 0 & 0 & A_{1,1}^{\lambda}-s I_{n_{1}} & B_{1,1}^{\lambda} & A_{1, s}^{\lambda} \\
0 & 0 & 0 & C_{1,1}^{\lambda} & D_{1,1}^{\lambda} & C_{1, s}^{\lambda}
\end{array}\right)
$$

Moreover, we have that $\rho\left(\mathbf{U}_{\mathbf{0}}, \mathbf{Y}\right)=\operatorname{card}\left(\mathbf{U}_{\mathbf{0}}\right)=q_{0}=$ $\operatorname{card}\left(\mathbf{S}^{\mathbf{o}}\left(\mathbf{U}_{\mathbf{0}}, \mathbf{Y}\right)\right)=p_{0}+n_{s}$ and so

$P_{1}(s) \stackrel{\text { def }}{=}\left(\begin{array}{ccc}A_{0,0}^{\lambda}-s I_{n_{0}} & A_{0, s}^{\lambda} & B_{0,0}^{\lambda} \\ A_{s, 0}^{\lambda} & A_{s, s}^{\lambda}-s I_{n_{s}} & B_{s, 0}^{\lambda} \\ C_{0,0}^{\lambda} & C_{0, s}^{\lambda} & D_{0,0}^{\lambda} \\ 0 & I_{n_{s}} & 0\end{array}\right)$ is a square ma-

trix. Matrix $P_{e}(s)$ has generically full column rank $\forall s \in \mathbb{C}$ iff $P_{1}(s)$ and $P_{2}(s)$ have both generically full column rank $\forall s \in \mathbb{C}$, where $P_{2}(s) \stackrel{\text { def }}{=}\left(\begin{array}{ccc}A_{1,1}^{\lambda}-s I_{n_{1}} & B_{1,1}^{\lambda} & A_{1, s}^{\lambda} \\ C_{1,1}^{\lambda} & D_{1,1}^{\lambda} & C_{1, s}^{\lambda}\end{array}\right)$.

Necessity and Sufficiency of Cond2 and Cond3:

for $P_{1}(s)$, we can apply Theorem 5.1 of (van der Woude, 2000) which states that the degree of the determinant of $P_{1}(s)$ is generically equal to $n_{0}+q_{0}-\left(\mu\left(\mathbf{U}_{\mathbf{0}}, \mathbf{S}^{\mathbf{o}}\left(\mathbf{U}_{\mathbf{0}}, \mathbf{Y}\right)\right)-\right.$ $\operatorname{card}\left(\mathbf{S}^{\mathbf{o}}\left(\mathbf{U}_{\mathbf{0}}, \mathbf{Y}\right)\right)$ ), with obviously $\mathbf{S}^{\mathbf{o}}\left(\mathbf{U}_{\mathbf{0}}, \mathbf{Y}\right)=\mathbf{Y}_{\mathbf{0}} \cup \mathbf{X}_{\mathbf{s}}$. This determinant is non-zero $\forall s \in \mathbb{C}$ iff its degree is equal to 0 and $g_{-} r a n k\left(P_{1}(0)\right)=n_{0}+q_{0}+n_{s}$. Yet, according to $\theta\left(\mathbf{X}_{\mathbf{0}} \cup \mathbf{U}_{\mathbf{0}}, \mathbf{X}_{\mathbf{1}} \cup \mathbf{U}_{\mathbf{1}}\right)=0$, the existence of a $v$-disjoint subgraph which covers $\mathbf{X}_{\mathbf{0}} \cup \mathbf{U}_{\mathbf{0}}$, with the requirements enounced in Cond2 and Cond3, is necessary and sufficient to ensure that $g_{-} \operatorname{rank}\left(P_{1}(0)\right)=n_{0}+q_{0}+n_{s}$ and $\mu\left(\mathbf{U}_{\mathbf{0}}, \mathbf{S}^{\mathbf{o}}\left(\mathbf{U}_{\mathbf{0}}, \mathbf{Y}\right)\right)=n_{0}+q_{0}+\operatorname{card}\left(\mathbf{S}^{\mathbf{o}}\left(\mathbf{U}_{\mathbf{0}}, \mathbf{Y}\right)\right)$, and so, to ensure that $P_{1}(s)$ has generically full column rank i.e. $g \_r a n k\left(P_{1}(s)\right)=n_{0}+q_{0}+n_{s}, \forall s \in \mathbb{C}$.

Sufficiency of condition Cond1: From Theorem 5.2 of (van der Woude, 2000), adapted to the observation context, we have:

If $P_{2}(s)$ has generically full column n-rank even after the deletion of an arbitrary row, then, generically, the greatest common divisor of all the $\left(n_{1}+q_{1}+n_{s}\right)^{\text {th }}$ order minors of $P_{2}(s)$ is a monomial in $s$ with a degree equal to: $n_{1}+q_{1}+n_{s}$ minus the maximum number of edges in the disjoint union of a linking of size $n_{s}+q_{1}$ from $\mathbf{U}_{\mathbf{1}} \cup \mathbf{X}_{\mathbf{s}}$ to $\mathbf{Y}$, an $\mathbf{Y}$-topped family and a cycle family in $\mathbf{X}_{\mathbf{1}}$.

Cond1 implies that $\rho\left(\mathbf{U}_{\mathbf{1}} \cup \mathbf{X}_{\mathbf{s}}, \mathbf{Y}_{\mathbf{1}}\right)=q_{1}+n_{s}$ and that the maximal number of edges in a disjoint union of a linking of size $n_{s}+q_{1}$ from $\mathbf{U}_{\mathbf{1}} \cup \mathbf{X}_{\mathbf{s}}$ to $\mathbf{Y}$, an $\mathbf{Y}$-topped family and a cycle family in $\mathbf{X}_{\mathbf{1}}$ is equal to $n_{1}+n_{s}+q_{1}$. Thus, if the hypothesis that generically $P_{2}(s)$ has full column $n$ rank even after the deletion of an arbitrary row, is satisfied then, generically, the greatest common divisor of all the $\left(n_{1}+q_{1}+n_{s}\right)^{t h}$ order minors of $P_{2}(s)$ is a monomial in $s$ with a degree equal to zero and so $P_{2}(s)$ has generically full column rank.

We will now show that the hypothesis that $P_{2}(s)$ generically has full column $n$-rank even after the deletion of an arbitrary row, is true. That is to say $g \_n$-rank $\left(P_{2, i}(s)\right)=n_{1}+n_{s}+q_{1}$, $\forall i=1, \ldots, n_{1}+q_{1}$, where $P_{2, i}(s)$, for $i=1, \ldots, n_{1}+q_{1}$, corresponds to matrix $P_{2}(s)$ after the deletion of its $i^{\text {th }}$ row. The deletion of the $i^{t h}$ row of $\left(A_{1,1}^{\lambda}-s I_{n_{1}} B_{1,1}^{\lambda} A_{1, s}^{\lambda}\right)$ in $P_{2}(s)$ is equivalent to the deletion of all the edges ending by $\mathbf{x}_{\mathbf{i}_{1}}$ in the digraph, where $x_{i_{1}}$ is the $i^{t h}$ component of the state subvector $X_{1}$. Yet, $\rho\left(\mathbf{U} \cup\left\{\mathbf{x}_{\mathbf{i}_{1}}\right\}, \mathbf{Y}\right)>\rho(\mathbf{U}, \mathbf{Y})$ and so $\rho\left(\mathbf{S}^{\mathbf{o}}(\mathbf{U}, \mathbf{Y}) \cup\left\{\mathbf{x}_{\mathbf{i}_{1}}\right\}, \mathbf{Y}\right)>\rho\left(\mathbf{S}^{\mathbf{o}}(\mathbf{U}, \mathbf{Y}), \mathbf{Y}\right)$. Moreover, from Lemma $5, \mathbf{S}^{\mathbf{o}}(\mathbf{U}, \mathbf{Y})=\mathbf{U}_{\mathbf{1}} \cup \mathbf{X}_{\mathbf{s}} \cup \mathbf{Y}_{\mathbf{0}}$. Knowing that $\mathbf{Y}_{\mathbf{1}}=\mathbf{Y} \backslash \mathbf{Y}_{\mathbf{0}}$, we have that $\rho\left(\mathbf{U}_{\mathbf{1}} \cup \mathbf{X}_{\mathbf{s}} \cup\left\{\mathbf{x}_{\mathbf{i}_{1}}\right\}, \mathbf{Y}_{\mathbf{1}}\right)>$ $\rho\left(\mathbf{U}_{\mathbf{1}} \cup \mathbf{X}_{\mathbf{s}}, \mathbf{Y}_{\mathbf{1}}\right)=q_{1}+n_{s}$. Then, $\rho\left(\mathbf{U}_{\mathbf{1}} \cup \mathbf{X}_{\mathbf{s}} \cup\left\{\mathbf{x}_{\mathbf{i}_{1}}\right\}, \mathbf{Y}_{\mathbf{1}}\right)=$ $q_{1}+n_{s}+1$. Applying results of (van der Woude, 2000), we have that for $i<n_{1}, g \_n$-rank $\left(P_{2, i}(s)\right)$ is equal to $n_{1}-1$ plus the maximal size of a $\mathbf{U}_{\mathbf{1}} \cup \mathbf{X}_{\mathbf{s}} \cup\left\{\mathbf{x}_{\mathbf{i}_{1}}\right\}-\mathbf{Y}_{\mathbf{1}}$ linking. Thus, $g \_n$-rank $\left(P_{2, i}(s)\right)=n_{1}+q_{1}+n_{s}$ for $i \leq n_{1}$.

The deletion of the $i^{t h}$ row of $\left(C_{1,1}^{\lambda} D_{1,1}^{\lambda} C_{1, s}^{\lambda}\right)$ in $P_{2}(s)$ is equivalent to the deletion of all edges ending by $\mathbf{y}_{\mathbf{i}_{1}}$ in the digraph, where $y_{i_{1}}$ is the $i^{\text {th }}$ component of out- 
put subvector $Y_{1}$. Yet, $\rho(\mathbf{U}, \mathbf{Y})=\rho\left(\mathbf{U}, \mathbf{Y} \backslash\left\{\mathbf{y}_{\mathbf{i}_{1}}\right\}\right)$. Thus, $\rho\left(\mathbf{S}^{\mathbf{o}}(\mathbf{U}, \mathbf{Y}), \mathbf{Y}\right)=\rho\left(\mathbf{U}_{\mathbf{1}} \cup \mathbf{X}_{\mathbf{s}} \cup \mathbf{Y}_{\mathbf{0}}, \mathbf{Y} \backslash\left\{\mathbf{y}_{\mathbf{i}_{1}}\right\}\right)$. As $\mathbf{Y}_{\mathbf{1}}=$ $\mathbf{Y} \backslash \mathbf{Y}_{\mathbf{0}}$, we have that $\rho\left(\mathbf{U}_{\mathbf{1}} \cup \mathbf{X}_{\mathbf{s}}, \mathbf{Y}_{\mathbf{1}} \backslash\left\{\mathbf{y}_{\mathbf{i}_{1}}\right\}\right)=q_{1}+n_{s}$. Applying results of (van der Woude, 2000), we have that $g \_n$-rank $\left(P_{2, i}(s)\right)$ for $n_{1}<i \leq n_{1}+p_{1}$, is equal to $n_{1}$ plus the maximal size of a $\mathbf{U}_{\mathbf{1}} \cup \overline{\mathbf{X}}_{\mathbf{s}}-\mathbf{Y}_{\mathbf{1}} \backslash\left\{\mathbf{y}_{\mathbf{i}_{1}}\right\}$ linking. So, for $n_{1}<i \leq n_{1}+p_{1}, g \_n$-rank $\left(P_{2, i}(s)\right)=n_{1}+q_{1}+n_{s}$. To summarize, Cond1 of Proposition 6 ensures that:

- the maximal number of edges in the disjoint union of a linking of size $n_{s}+q_{1}$ from $\mathbf{U}_{\mathbf{1}} \cup \mathbf{X}_{\mathbf{s}}$ to $\mathbf{Y}$, an $\mathbf{Y}$-topped family and a cycle family in $\mathbf{X}_{\mathbf{1}}$ is equal to $n_{1}+n_{s}+q_{1}$;

- $P_{2}(s)$ has generically full column $n$-rank, even after the deletion of an arbitrary row.

Therefore, the greatest common divisor of all the $\left(n_{1}+\right.$ $\left.q_{1}+n_{s}\right)^{t h}$ order minors of $P_{2}(s)$ is generically a monomial in $s$ with a degree equal to zero. As $g_{-} \operatorname{rank}\left(P_{2}(0)\right)=$ $n_{1}+n_{s}+q_{1}$, then $P_{2}(s)$ has generically full column rank $\forall s \in \mathbb{C}$.

Thus, conditions of Proposition 6 are necessary and sufficient to ensure that $\forall s \in \mathbb{C}, P_{e}(s)$ has generically full column rank. Then, g_rank $(P(s))=n+q, \forall s \in \mathbb{C}$.

We can deduce from Proposition 6 other simpler graphic conditions:

Corollary $7 \Sigma_{\Lambda}$ is generically state and input observable iff

Ca. in its associated digraph $\mathcal{G}\left(\Sigma_{\Lambda}\right)$, there exists a vdisjoint subgraph $\mathcal{S}_{\mathcal{G}}$ which covers $\mathbf{X} \cup \mathbf{U}$;

Cb. $\quad \mathbf{X}_{\mathbf{0}} \cup \mathbf{U}_{\mathbf{0}} \subseteq V_{e s s}\left(\mathbf{U}_{\mathbf{0}}, \mathbf{Y}_{\mathbf{0}} \cup \mathbf{X}_{\mathbf{s}}\right)$.

Proof: First note that condition Ca is the same as Cond1 in Proposition 6. So, we have only to prove hereafter that condition $\mathbf{C b}$ of Corollary 7 is equivalent to conditions Cond2 and Cond 3 in Proposition 6.

Necessity: If condition $\mathbf{C b}$ is not satisfied then $\operatorname{card}\left(\mathbf{X}_{\mathbf{0}} \cup\right.$ $\overline{\left.\mathbf{U}_{\mathbf{0}}\right)>\mu}\left(\mathbf{U}_{\mathbf{0}}, \mathbf{S}^{\mathbf{o}}\left(\mathbf{U}_{\mathbf{0}}, \mathbf{Y}\right)\right)-\rho\left(\mathbf{U}_{\mathbf{0}}, \mathbf{S}^{\mathbf{o}}\left(\mathbf{U}_{\mathbf{0}}, \mathbf{Y}\right)\right)$. In this case, we cannot cover all the elements of $\mathbf{X}_{\mathbf{0}} \cup \mathbf{U}_{\mathbf{0}}$ with paths of total length $\mu\left(\mathbf{U}_{\mathbf{0}}, \mathbf{S}^{\mathbf{o}}\left(\mathbf{U}_{\mathbf{0}}, \mathbf{Y}\right)\right)-\operatorname{card}\left(\mathbf{S}^{\mathbf{o}}\left(\mathbf{U}_{\mathbf{0}}, \mathbf{Y}\right)\right)$. Thus, since no cycle can be used to cover $\mathbf{X}_{\mathbf{0}} \cup \mathbf{U}_{\mathbf{0}}$, there cannot exist a $v$-disjoint subgraph which covers $\mathbf{X}_{\mathbf{0}} \cup \mathbf{U}_{\mathbf{0}}$ and which satisfies Cond2 and Cond3.

Sufficiency: Assume that $\mathbf{C a}$ is satisfied. Condition $\mathbf{C b}$ implies, on the one hand, that $\rho\left(\mathbf{U}_{\mathbf{0}}, \mathbf{Y}\right)=\operatorname{card}\left(\mathbf{U}_{\mathbf{0}}\right)$ and on the other hand, that card $\left(\mathbf{X}_{\mathbf{0}} \cup \mathbf{U}_{\mathbf{0}}\right)=\mu\left(\mathbf{U}_{\mathbf{0}}, \mathbf{S}^{\mathbf{o}}\left(\mathbf{U}_{\mathbf{0}}, \mathbf{Y}\right)\right)-$ $\rho\left(\mathbf{U}_{\mathbf{0}}, \mathbf{S}^{\mathbf{o}}\left(\mathbf{U}_{\mathbf{0}}, \mathbf{Y}\right)\right)$. So, all the $v$-disjoint subgraphs which cover $\mathbf{X}_{\mathbf{0}} \cup \mathbf{U}_{\mathbf{0}}$ are constituted by a maximum $\mathbf{U}_{\mathbf{0}}-\mathbf{X}_{\mathbf{s}} \cup \mathbf{Y}_{\mathbf{0}}$ linking of a minimal length. Therefore, all $v$-disjoint subgraphs covering $\mathbf{X} \cup \mathbf{U}$ contain a maximum $\mathbf{U}_{\mathbf{0}}-\mathbf{X}_{\mathbf{s}} \cup \mathbf{Y}_{\mathbf{0}}$ linking of a minimal length, which covers $\mathbf{X}_{\mathbf{0}} \cup \mathbf{U}_{\mathbf{0}}$ and so cannot contain any cycles on $\mathbf{X}_{\mathbf{0}} \cup \mathbf{X}_{\mathbf{s}}$. This is sufficient to ensure that conditions Cond2 and Cond3 are satisfied. $\triangle$

Finally, note that conditions of Corollary 7 generalize explicitly the well-known state observability conditions for linear systems without unknown inputs. Indeed, if $\mathbf{U}=\emptyset, \mathbf{C b}$ is equivalent to $\mathbf{X}_{\mathbf{0}}=\emptyset$ and so, each state component is the begin vertex of an $\mathbf{Y}$-topped path and $\mathbf{C a}$ is equivalent to the existence of disjoint $\mathbf{Y}$-topped paths and cycles, which cover X (Dion et al., 2003).

\section{Conclusion}

In this paper, we propose a graph-theoretic tool to analyze the state and input generic observability for structured linear systems. Necessary and sufficient conditions for state and unknown input generic observability are given and expressed in graphic terms. These intrinsic conditions need few information about the system. Moreover, they are easy to check by means of well-known combinatorial techniques. Indeed, from a computational point of view, to check the first condition of Corollary 7, we use the Bipmatch method (Micali and Vazirani, 1980), which complexity order of algorithms is $O\left(M \times N^{0.5}\right)$, where $M=n^{2}+n q+n p+p q$ is the number of edges and $N=n+q+p$ is the number of vertices in the digraph. Furthermore, condition $\mathbf{C b}$ can be checked using depth search algorithms. These algorithms have a complexity order $O(M \times N)$. Thus, the global complexity of our method is $O\left(n^{3}\right)$. This is why our approach is particularly suited for large-scale systems.

\section{References}

Basile, G. \& Marro, G. (1973). A new characterization of some structural properties of linear systems: unknown-input observability, invertibility and functional controllability. International Journal of Control, 17(5), 931-943.

Boukhobza, T., Hamelin, F. \& Sauter, D. (2006). Observability of structured linear systems in descriptor form: a graph-theoretic approach. Automatica, 42(6), 629-635.

Chu, D. (2000). Disturbance decoupled observer design for linear timeinvariant systems: a matrix pencil approach. IEEE Transactions on Automatic Control, 45(8), 1569-1575.

Chu, D. \& Mehrmann, V. (1999). Disturbance decoupled observer design for descriptor systems. System \& Control Letters, 38(1), 37-48.

Dion, J-M., Commault, C., \& Van der Woude, J. (2003). Generic properties and control of linear structured systems: A survey. Automatica, 39(7), 1125-1144.

Hou, M. \& Müller, P. C. (1992). Design of observers for linear systems with unknown input. IEEE Transactions on Automatic Control, 37(4), 871-875.

Hou, M. \& Müller, P. C. (1999). Causal observability of descriptor systems. IEEE Transactions on Automatic Control, 44(1), 158-163.

Hou, M. \& Patton, R. J. (1998). Input observability and input reconstruction. Automatica, 34(6), 789-794.

Hou, M., Pugh, A. C. \& Müller, P. C. (1999). Disturbance decoupled functionnal observer. IEEE Transactions on Automatic Control, 44, 382-386.

Koenig, D. (2005). Unknown input proportional multiple-integral observer design for linear descriptor systems: application to state and fault estimation. IEEE Transactions on Automatic Control, 50(2), 212217.

Micali, S. \& Vazirani, V. V.(1980). An $O\left(\left|V^{1 / 2} E\right|\right)$ algorithm for finding maximum matching in general graphs. In Proceedings of the $21^{\text {st }}$ Annual Symposium on the Foundations of Computer Science, pages 17-27.

Murota, K. (1987). System Analysis by Graphs and Matroids. SpringerVerlag. New York, U.S.A.

Reinschke, K. J. (1988). Multivariable Control. A Graph Theoretic Approach.. Springer-Verlag. New York, U.S.A.

Trentelman, H. L., Stoorvogel, A. A. \& Hautus, M. (2001). Control Theory for Linear Systems. Springer, London, U.K.,

Trinh, H. \& Ha, Q. (2000). Design of linear functional observers for linear systems with unknown inputs. International Journal of Systems Science, 31(6), 741-749.

Tsui, C. C. (1996). A new design approach to unknown input observers. IEEE Transactions on Automatic Control, 41(3), 464-468.

van der Woude, J. W. (2000). The generic number of invariant zeros of a structured linear system. SIAM Journal of Control and Optimization, $38(1), 1-21$. 